\title{
A variational model of fracture for tearing brittle thin sheets
}

\author{
Bin Li ${ }^{\mathrm{a}, \mathrm{b}}$, Daniel Millán ${ }^{\mathrm{a}, \mathrm{c}}$, Alejandro Torres-Sánchez ${ }^{\mathrm{a}}$, Benoît Roman ${ }^{\mathrm{d}}$, Marino Arroyo ${ }^{\mathrm{a}, *}$ \\ ${ }^{a}$ LaCàN, Universitat Politècnica de Catalunya-BarcelonaTech (UPC), Barcelona, Spain \\ ${ }^{b}$ Sorbonne Université, CNRS, Institut Jean Le Rond d'Alembert, UMR 7190, F-75005, Paris, France \\ ${ }^{c}$ CONICET $\&$ Facultad de Ciencias Aplicadas a la Industria, Universidad Nacional de Cuyo, San Rafael, Argentina \\ ${ }^{d}$ Laboratoire de Physique et Mécanique des Milieux Hétérogènes (PMMH), CNRS, ESPCI Paris, \\ PSL Research University, Sorbonne Université, Univ. Paris Diderot. Paris, France
}

\begin{abstract}
Tearing of brittle thin elastic sheets, possibly adhered to a substrate, involves a rich interplay between nonlinear elasticity, geometry, adhesion, and fracture mechanics. In addition to its intrinsic and practical interest, tearing of thin sheets has helped elucidate fundamental aspects of fracture mechanics including the mechanism of crack path selection. A wealth of experimental observations in different experimental setups is available, which has been often rationalized with insightful yet simplified theoretical models based on energetic considerations. In contrast, no computational method has addressed tearing in brittle thin elastic sheets. Here, motivated by the variational nature of simplified models that successfully explain crack paths in tearing sheets, we present a variational phase-field model of fracture coupled to a nonlinear Koiter thin shell model including stretching and bending. We show that this general yet straightforward approach is able to reproduce the observed phenomenology, including spiral or power-law crack paths in free standing films, or converging/diverging cracks in thin films adhered to negatively/positively curved surfaces, a scenario not amenable to simple models. Turning to more quantitative experiments on thin sheets adhered to planar surfaces, our simulations allow us to examine the boundaries of existing theories and suggest that homogeneous damage induced by moving folds is responsible for a systematic discrepancy between theory and experiments. Thus, our computational approach to tearing provides a new tool to understand these complex processes involving fracture, geometric nonlinearity and delamination, complementing experiments and simplified theories.
\end{abstract}

Keywords: variational model, tearing, fracture, thin sheets, subdivision surface.

\section{Introduction}

Thin elastic sheets are very common in nature and technology. In addition to an in-plane mode of fracture, thin sheets exhibit tearing, a situation in which cracks propagate driven by out-of-plane loading. Tearing a thin sheet is a very common experience in our daily life when we peel a piece of fruit or open a package. We lack, however, a complete theoretical understanding of this phenomenon, which challenges classical theories/of fracture. In classical fracture mechanics, various crack path selection criteria have been successful in predicting crack propagation in bulk brittle materials, including the maximum hoop stress criterion (Erdogan and Sih, 1963), the principle of local symmetry (Cotterell, 1965; Goldstein and Salganik, 1974), the minimum strain energy density (Sih, 1974), or maximum energy release rate (Wu, 1978; Palaniswamy and Knauss, 1978). While these different criteria are very similar, or even equivalent, for bulk isotropic materials, it is far from obvious how to generalize some of them to a brittle, possibly anisotropic, thin sheet (Takei et al., 2013; Roman, 2013; Ibarra et al., 2016). For instance, the principle of local symmetry

\footnotetext{
* Corresponding author

Email address: marino.arroyo@upc.edu (Marino Arroyo)
} 
relies on stress intensity factors (SIFs). The study of the stress field around a crack tip in linear elastic thin plates (Williams, 1961; Sih et al., 1962; Zehnder and Viz, 2005) and Kirchhoff-Love shells (Folias, 1977), has identified, in addition to the usual in-plane SIFs $K_{\mathrm{I}}$ and $K_{\mathrm{II}}$, two additional SIFs $k_{1}$ and $k_{2}$, which correspond to a symmetric bending mode and antisymmetric twisting and shearing mode. How to extend the principle of local symmetry to this setting is not obvious (Roman, 2013). Furthermore, tearing is typically characterized by large geometric nonlinearity (Hamm et al., 2008; Bayart et al., 2010, 2011; Kruglova et al., 2011; Takei et al., 2013; Roman, 2013; Ibarra et al., 2016), and therefore it is not clear whether the crack tip fields of the linear theory characterized by the SIFs are meaningful (Hui et al., 1998) or if and how they determine crack propagation (Cohen and Procaccia, 2010). There have been attempts to develop theories that explain tearing in thin sheets including geometric nonlinearity (Cohen and Procaccia, 2010), which have focused on characterizing the stress state in the vicinity of the crack tip and have invoked a generalized principle of local symmetry. This reference captures some qualitative features of crack propagation such as convergent cracks in three-flap tearing tests, but fails to describe the power-law geometry of the crack path (Bayart et al., 2010, 2011).

The interplay between geometry, surface energy, stretching and bending deformation leads to non-trivial and rich behaviors (Bayart et al., 2010, 2011; Takei et al., 2013; Brau, 2014; Ibarra et al., 2016), particularly when the thin film is adhesively coupled to a flat (Hamm et al., 2008) or curved (Kruglova et al., 2011) substrate. The complexity of these problems restricts analytical approximate solutions to very simplified settings (e.g. planar sheets) and specific parameter regimes (e.g. inextensible limit) (Hamm et al., 2008; Roman, 2013; Brau, 2014). Simple energetic models in these references have been remarkably successful in explaining almost quantitatively nontrivial observations such as the dependence of crack path on interfacial adhesion (Hamm et al., 2008; Roman, 2013), peeling angle (Bayart et al., 2011; Roman, 2013; Brau, 2014), or anisotropy in the fracture surface energy (Takei et al., 2013; Ibarra et al., 2016). However, a general modeling approach to tearing, capable of examining in detail the mechanics of tearing in general geometries and arbitrary material parameter regimes has beenlacking.

The success of restricted variational models suggests that the general variational approach to brittle fracture may provide a unifying and general framework extending from bulk brittle fracture to materials with strongly anisotropic surface energy (Li et al., 2015) or to tearing of brittle thin elastic sheets. The variational approach to brittle fracture proposed by Francfort and Marigo (1998), which formulates the crack initiation and quasi-static evolution in terms of the minimization of a Griffith-like energy functional consisting of the elastic energy and surface energy of a cracked body. This theory was subsequently regularized into a phasefield or gradient damage model suitable for numerical calculations (Bourdin et al., 2000; Bourdin, 2007), which converges to the sharp variational theory of brittle fracture (Bourdin et al., 2008). In these models, the complexity of tracking and evolving cracks is addressed by introducing an additional field describing cracks in a smeared way, which needs to be solved for using a partial differential equation coupled to the equations of elasticity. Subsequently, Pham et al. (2011); Pham and Marigo (2013); Marigo et al. (2016) interpreted it as a non-local gradient damage model and proposed a general class of variational gradient damage models that Gamma-converge to Griffith brittle fracture theory (Braides, 1998) and have some advantages from numerical and theoretical standpoints (Pham et al., 2011). These works have prompted further developments extending the original approach to account for fracture in piezoelectric and ferroelectric materials (Abdollahi and Arias, 2012), fracture in rubbery polymers (Miehe and Schänzel, 2014), complex crack patterns induced by thermal shocks (Maurini et al., 2013; Bourdin et al., 2014; Sicsic et al., 2014), thin film fracture and delamination (Mesgarnejad et al., 2013; Baldelli et al., 2013, 2014), hydraulic fracture (Wilson and Landis, 2016), and fracture in linear (Amiri et al., 2014; Kiendl et al., 2016) and nonlinear thin shells (Reinoso et al., 2017; Millán et al., 2018).

To systematically explore tearing of thin films, we develop here a model coupling a geometrically exact Koiter thin shell model capturing stretching and bending elasticiy, fracture, and adhesion to a substrate, see Section 2. Brittle fracture is modeled using the variational approach to fracture (Bourdin et al., 2008). The delamination of thin sheets adhered to substrates is modeled with a cohesive model (Xu and Needleman, 1994). We numerically implement the model with subdivision surface finite elements (Cirak et al., 2000), see Section 3. In Section 4 we demonstrate that this modeling approach is capable of reproducing tearing behaviors involving complex crack paths observed experimentally in a variety of assays. To our knowledge, 
ours are the first computational results capturing the phenomenology of tearing. We quantitatively examine tearing of thin sheets adhered to flat substrates in the light of previous theoretical predictions and experiments, delineating the boundaries of our understanding of tearing.

\section{Theoretical model}

\subsection{Nonlinearly elastic model of thin sheets}

We model thin elastic sheets with a geometrically exact nonlinear thin shell formulation sometimes referred to as the nonlinear Koiter shell model (Ciarlet, 2005). We provide below a succinct description of this theory. More details about the justification of this theory from three-dimensional elasticity can be found in Ciarlet (2005), Steigmann (2013), and references therein.

In this theory, the middle surface of the undeformed shell $\Omega_{0}$ is parametrized with a mapping $\varphi_{0}$ from a parametric domain $\mathscr{A} \subset \mathbb{R}^{2}$ into $\mathbb{R}^{3}$. We describe $\mathscr{A}$ with Cartesian coordinates $\left(\xi^{1}, \xi^{2}\right)$. Likewise, another mapping $\varphi$ describes the deformed middle surface $\Omega$, see Figure 1. These mappings induce curvilinear coordinates on the undeformed and deformed surfaces. We use Greek indices to denote these curvilinear coordinates, a comma before an index denotes partial differentiation, subscripts refer to covariant components, and superscripts denote contravariant components.

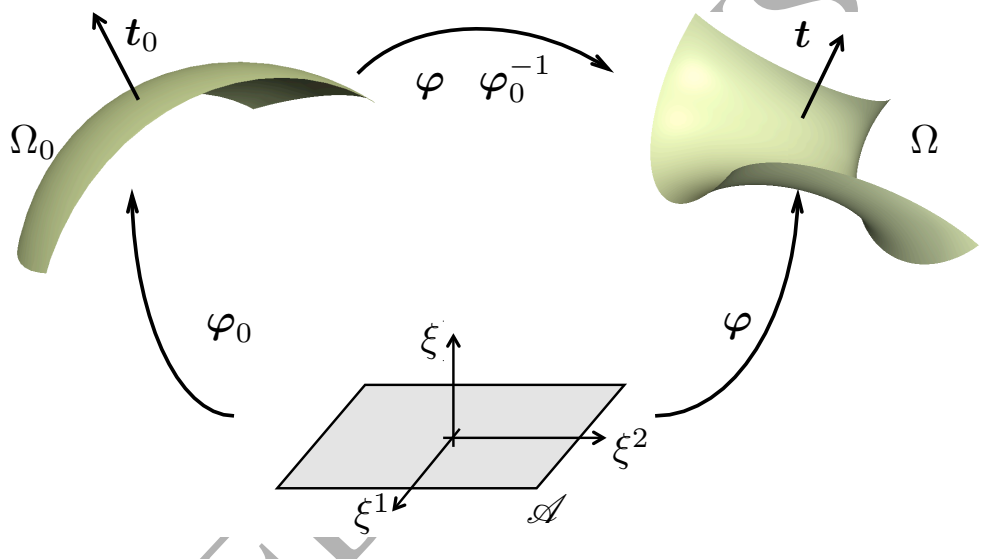

Figure 1: Reference, deformed and parametric configurations of the middle surface of thin shell.

The area element of the deformed middle surface can be computed as $d \Omega=\bar{j} d \xi^{1} d \xi^{2}$, where $\bar{j}=$ $\left|\varphi_{, 1} \times \varphi_{, 2}\right|$, and the unit normal is $\boldsymbol{t}=\left(\boldsymbol{\varphi}_{, 1} \times \varphi_{, 2}\right) / \bar{j}$. Analogous objects can be defined on the undeformed middle surface. The membrane strain tensor is defined as $\varepsilon_{\alpha \beta}=\frac{1}{2}\left(\boldsymbol{\varphi}_{, \alpha} \cdot \boldsymbol{\varphi}_{, \beta}-\boldsymbol{\varphi}_{0, \alpha} \cdot \boldsymbol{\varphi}_{0, \beta}\right)$, which measures changes in the in-plane metric tensor or first fundamental form (Do Carmo, 1976). The bending strain tensor, measuring changes in the second fundamental form, is given by $\rho_{\alpha \beta}=\boldsymbol{\varphi}_{, \alpha} \cdot \boldsymbol{t}_{, \beta}-\boldsymbol{\varphi}_{0, \alpha} \cdot \boldsymbol{t}_{0, \beta}$.

In Koiter's thin shell model, the elastic strain energy is expressed exclusively in terms of the kinematics of the middle surface, that is the mapping $\varphi$, and is written as

$$
\Pi_{\mathrm{ela}}[\varphi]=\int_{\Omega_{0}} W(\varepsilon, \boldsymbol{\rho}) d \Omega_{0}
$$

where $W$ is the strain energy density per unit undeformed surface, which for an isotropic material and a shell of thickness $t$ can be expressed as

$$
W(\varepsilon, \boldsymbol{\rho})=\frac{1}{2} \mathbb{C}^{\alpha \beta \gamma \delta}\left(t \varepsilon_{\alpha \beta} \varepsilon_{\gamma \delta}+\frac{t^{3}}{12} \rho_{\alpha \beta} \rho_{\gamma \delta}\right),
$$

with

$$
\mathbb{C}^{\alpha \beta \gamma \delta}=\frac{E}{\left(1-\nu^{2}\right)}\left[\nu a_{0}^{\alpha \beta} a_{0}^{\gamma \delta}+\frac{1}{2}(1-\nu)\left(a_{0}^{\alpha \gamma} a_{0}^{\beta \delta}+a_{0}^{\alpha \delta} a_{0}^{\beta \gamma}\right)\right]
$$


where $\left(a_{0}\right)_{\alpha \beta}=\boldsymbol{\varphi}_{0, \alpha} \cdot \boldsymbol{\varphi}_{0, \beta}$ are the convected components of the surface metric tensor, $\left(a_{0}\right)^{\alpha \beta}$ are defined by the relation $a_{0}^{\alpha \gamma}\left(a_{0}\right)_{\gamma \beta}=\delta_{\beta}^{\alpha}, E$ is the Young's modulus and $\nu$ is Poisson's ratio.

The membrane and bending stress resultants in this theory are given by

$$
n^{\alpha \beta}=\frac{\partial W}{\partial \varepsilon_{\alpha \beta}}=t \mathbb{C}^{\alpha \beta \gamma \delta} \varepsilon_{\gamma \delta}, \quad m^{\alpha \beta}=\frac{\partial W}{\partial \rho_{\alpha \beta}}=\frac{t^{3}}{12} \mathbb{C}^{\alpha \beta \gamma \delta} \rho_{\gamma \delta} .
$$

In the numerical implementation, it is convenient to resort to Voigt's notation, denoted by $\{\cdot\}$, which exploits the symmetry of the tensors involved in the theory. With this notation, we have

$$
\begin{aligned}
& \{\boldsymbol{n}\}=\left(\begin{array}{c}
n^{11} \\
n^{22} \\
n^{12}
\end{array}\right)=t\{\mathbb{C}\}\{\boldsymbol{\varepsilon}\}, \quad\{\boldsymbol{m}\}=\left(\begin{array}{c}
m^{11} \\
m^{22} \\
m^{12}
\end{array}\right)=\frac{t^{3}}{12}\{\mathbb{C}\}\{\boldsymbol{\rho}\}, \\
& \{\boldsymbol{\varepsilon}\}=\left(\begin{array}{c}
\varepsilon_{11} \\
\varepsilon_{22} \\
2 \varepsilon_{12}
\end{array}\right), \quad\{\boldsymbol{\rho}\}=\left(\begin{array}{c}
\rho_{11} \\
\rho_{22} \\
2 \rho_{12}
\end{array}\right)
\end{aligned}
$$

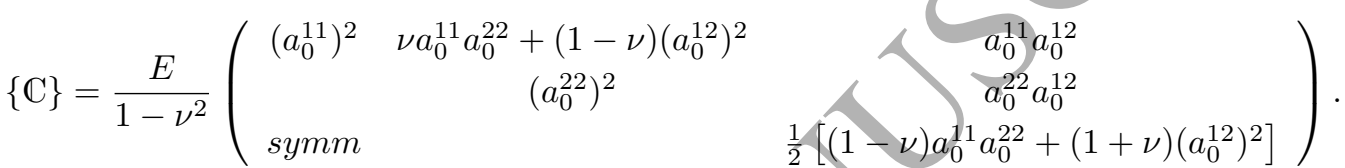

and

Using this notation and referring the integral to the referential domain, the elastic energy can be written as

$$
\Pi_{\mathrm{ela}}[\boldsymbol{\varphi}]=\int_{\mathscr{A}} \frac{1}{2}\left(t\{\varepsilon\}^{T}\{\mathbb{C}\}\{\varepsilon\}+\frac{t^{3}}{12}\{\boldsymbol{\rho}\}^{T}\{\mathbb{C}\}\{\boldsymbol{\rho}\}\right) \bar{j}_{0} d \xi^{1} d \xi^{2} .
$$

\subsection{Phase-field approximation of brittle fracture}

In the variational approach to brittle fracture proposed by Francfort and Marigo (1998), the crack initiation and quasi-static evolution are the natural results of the minimization of a Griffith-like energy functional defined as the sum of the elastic energy and the surface energy of the cracked body. The minimization has to be taken among all the kinematically admissible displacements and admissible crack sets, and subject to Dirichlet boundary conditions and an irreversibility condition to avoid unphysical healing of cracks. This theory has been subsequently regularized into a phase-field or gradient damage models, suitable for numerical calculations (Bourdin et al., 2000; Bourdin, 2007; Bourdin et al., 2008), and which converge to the sharp variational theory of brittle fracture (Bourdin et al., 2008). These and related models (Pham et al., 2011; Pham and Marigo, 2013) have been studied in detail in bulk materials and only barely explored in linear thin shells (Amiri et al. 2014, Kiendl et al., 2016).

In the regularized approximation of brittle fracture, cracks are represented by a phase-field variable (scalar order parameter) $\phi$, which is 0 inside a cracked zone, 1 away from the crack, and changes from 0 to 1 smoothly. In the present setting, we choose to describe $\phi$ as a field on the middle surface of the undeformed shell $\Omega_{0}$ only, implicitly assuming that the phase-field is constant across the thickness of thin sheet. Physically, this means that our model rules out partial cracking through the thickness, which is reasonable to model very thin shells but may not be adequate for thicker shells progressively cracking under bending. The model cannot resolve effects that may depend on the structure of the crack front through-thethickness. Despite these potential limitations, we will explore such a model, where the phase-field couples to the elastic energy through the modified elastic energy functional

$$
\Pi_{\mathrm{ela}}[\boldsymbol{\varphi}, \phi]=\int_{\Omega_{0}} \phi^{2} W(\varepsilon, \boldsymbol{\rho}) d \Omega_{0} .
$$

The other ingredient in a phase-field model of brittle fracture is a functional depending on $\phi$ approximating the crack length. In a finite deformation setting, it is natural to consider the length of the crack in the 
undeformed configuration. We consider a recently proposed higher-order phase-field model (Borden et al., 2014), which here needs to be formulated in the curved two-dimensional middle surface of the thin shell in its undeformed configuration (Millán et al., 2018) as

$$
\Pi_{\text {frac }}[\phi]=\int_{\Omega_{0}} G_{\mathrm{c}} t\left[\frac{(\phi-1)^{2}}{4 \kappa}+\frac{\kappa}{2}\left|\nabla_{\mathbf{s}} \phi\right|^{2}+\frac{\kappa^{3}}{4}\left(\Delta_{\mathbf{s}} \phi\right)^{2}\right] d \Omega_{0},
$$

where $G_{\mathrm{c}}$ is the critical energy release rate and $\nabla_{\mathrm{s}}$ and $\Delta_{\mathrm{s}}$ are the surface gradient and Laplacian operators in the undeformed middle surface. The metric tensor of this surface is given by $a_{\alpha \beta}=\varphi_{0, \alpha} \cdot \varphi_{0, \beta}$ and its contravariant components are given by the relation $a^{\alpha \gamma} a_{\gamma \beta}=\delta_{\beta}^{\alpha}$. Then, the expressions involving surface operators can be computed as

$$
\left|\nabla_{\mathbf{s}} \phi\right|^{2}=a^{\alpha \beta} \phi_{, \alpha} \phi, \beta
$$

and

where the comma denotes partial differentiation and

$$
\Delta_{\mathbf{s}} \phi=a^{\alpha \beta} \phi_{, \alpha \beta}-a^{\alpha \beta} \phi_{, \gamma} \Gamma_{\alpha \beta}^{\gamma},
$$

$$
\Gamma_{\alpha \beta}^{\gamma}=\frac{a^{\gamma \mu}}{2}\left(\frac{\partial a_{\alpha \mu}}{\partial \xi^{\beta}}+\frac{\partial a_{\beta \mu}}{\partial \xi^{\alpha}}-\frac{\partial a_{\alpha \beta}}{\partial \xi^{\mu}}\right)
$$

are the Christoffel symbols of the second kind (Marsden and Hughes, 1983). The regularization parameter $\kappa$ has units of length and dictates the width of the smeared crack. A finite value of $\kappa$ is necessary for the numerical simulations and needs to be resolved by the mesh.

We discuss next our motivation to use this higher-order phase field model of fracture instead of the standard second order model involving only up to first derivatives of $\phi$ in the fracture energy. Our choice allows us to use the same basis functions to discretize $\varphi$ and $\phi$. Indeed, because the Koiter model involves the second fundamental form, a direct Galerkin method requires smooth basis functions for $\varphi$, which in the present work are subdivision spline-like approximants. However, as shown in Borden et al. (2014); Amiri et al. (2016), if smooth basis functions are used to approximate $\phi$ in combination with the standard second order fracture energy, which exhibits solutions with discontinuous derivatives, then very poor numerical convergence is obtained. In contrast, the higher-order surface energy in Eq. (10) leads to smooth solutions for the phase field, which are very accurately approximated with higher convergence rates using smooth basis functions. Thus, we choose to treat on an equal footing the elastic and the fracture functionals: each of these functionals requires that the fields $\varphi$ and $\phi$ have second order square integrable derivatives, a condition which is numerically dealt with using smooth subdivision basis functions. Recent mathematical results have substantiated the higher order fracture energy used here, by showing that it is an elliptic approximation of the Mumford-Shah functional in the sense of Gamma-convergence (Burger et al., 2015). It is noteworthy that this fourth-order model is a particular instance of the extended Cahn-Hilliard model for fracture developed in Li et al. (2015).

\subsection{Adhesion energy between a thin sheet and a substrate}

We model the adhesive interaction between thin shells and rigid substrates by cohesive zone model based on an exponential potential (Xu and Needleman, 1994). In this model, the adhesion energy is expressed as

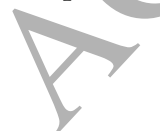

$$
\Pi_{\mathrm{adh}}[\varphi]=\int_{\Omega_{0}} \Gamma\left[1-\left(1+\frac{\Delta_{\mathrm{n}}}{\delta_{\mathrm{n}}}\right) \exp \left(-\frac{\Delta_{\mathrm{n}}}{\delta_{\mathrm{n}}}-\frac{\Delta_{\mathrm{t}}^{2}}{\delta_{\mathrm{t}}^{2}}\right)\right] d \Omega_{0},
$$

where the $\Gamma$ is interfacial adhesion energy per unit area, $\delta_{\mathrm{n}}$ and $\delta_{\mathrm{t}}$ are characteristic length-scales, and $\Delta_{\mathrm{n}}$ and $\Delta_{\mathrm{t}}$ are the normal and tangential components of the displacement jump across the interface. For a thin sheet adhered to a curved surface, $\Delta_{\mathrm{n}}$ and $\Delta_{\mathrm{t}}$ are computed by projecting the displacement of the middle surface $\boldsymbol{u}=\boldsymbol{\varphi}-\boldsymbol{\varphi}_{0}$ along the normal and tangential directions, $\Delta_{\mathrm{n}}=\boldsymbol{u} \cdot \boldsymbol{n}$ and $\Delta_{\mathrm{t}}=|\boldsymbol{u}-(\boldsymbol{u} \cdot \boldsymbol{n}) \boldsymbol{n}|$. To avoid interpenetration of the thin sheet into a rigid substrate, the condition $\Delta_{n} \geq 0$ should be imposed. Otherwise, this model just limits the extent of interpenetration with an elastic penalty. In all the examples 
presented later, in which sheets are torn away from the support, interpenetration into the substrate was very small even though the inequality constraint was not enforced. We also note that this model describes decohesion thanks to a non-convex energy landscape, which defines bound and unbound states under stress. It is thus reversible and does not preclude the formation of an adherent interface if a free-standing thin sheet is brought in close proximity of a substrate.

\subsection{Phase-field model of fracture in brittle adhesive thin sheets}

Collecting all the ingredients in the previous sections, the total energy of a possibly fractured adhesive thin sheets is

$$
\Pi_{\text {tot }}[\varphi, \phi]=\Pi_{\text {frac }}[\phi]+\Pi_{\mathrm{ela}}[\varphi, \phi]+\Pi_{\mathrm{adh}}[\boldsymbol{\varphi}] .
$$

The minimization of functional Eq.(15) with respect to both $\varphi$ and $\phi$, subject to Dirichlét boundary condition and to irreversibility of cracks provides a computable approximation of the generalized Griffith's brittle fracture model for geometrically nonlinear thin and adhesive shells.

\section{Numerical implementation}

Because the total energy involves second-order derivative of both the shell deformation $\varphi$ and of the phase-field $\phi$, a $C^{1}$ continuous approximation scheme is necessary to apply a straightforward Galerkin discretization approach. In our previous work ( $\mathrm{Li}$ et al., 2015), we resorted to smooth meshfree basis functions. Here, we use subdivision surface finite elements (Cirak et al., 2000; Cirak and Ortiz, 2001) with boundary control (Biermann et al., 2000; Cirak and Long, 2011) to approximate the deformation $\varphi$ and the phase-field $\phi$. We follow a total Lagrangian approach, with the same parameter space and basis functions for the undeformed and deformed configurations. Let $\varphi_{0}^{e}$ be the undeformed configuration mapping of the middle surface restricted to element $e$ and defined over the parametric space $\mathcal{A}^{e}$ of this element. It is numerically represented as

$$
\varphi_{0}^{e}\left(\xi^{1}, \xi^{2}\right)=\sum_{a=1}^{\mathcal{N}} B_{a}\left(\xi^{1}, \xi^{2}\right) \varphi_{0 a},
$$

where $B_{a}\left(\xi^{1}, \xi^{2}\right)$ are subdivision surfaces basis functions of that patch, $\mathcal{N}^{e}$ is the number of nodes contributing to element $e$, and $\varphi_{0 a}$ is the position in three-dimensional space of the $a$-th control point contributing to the approximation in element $e$ and defining the undeformed shell middle surface. Similarly, the deformed configuration and the phase-field are discretized as

$$
\varphi^{e}\left(\xi^{1}, \xi^{2}\right)=\sum_{a=1}^{\mathcal{N}^{e}} B_{a}\left(\xi^{1}, \xi^{2}\right) \varphi_{a}
$$

and

$$
\phi^{e}\left(\xi^{1}, \xi^{2}\right)=\sum_{a=1}^{\mathcal{N}^{e}} B_{a}\left(\xi^{1}, \xi^{2}\right) \phi_{a} .
$$

Inserting Eqs. $(17,18)$ into Eq. $(15)$, we obtain a total energy function depending on nodal variables $\boldsymbol{\varphi}_{a}$ and $\phi_{a}$. We minimize this energy with respect to deformation and phase-field degrees of freedom following the alternate minimization algorithm developed in Bourdin (2007); Bourdin et al. (2008). At each load increment, the energy is first minimized with respect to $\varphi_{a}$ freezing the phase-field and using Newton's method combined with line-search (Millán et al., 2013), and then minimized with respect to $\phi_{a}$ freezing the deformation, which results in a linear algebraic system. This procedure is iterated until convergence reached. We use the approach by Miehe et al. (2010) to approximate the irreversibility condition, which stores the maximum strain energy density $W$ achieved in history at each Gauss point and replaces $W$ by this history field in the minimization problem to solve for the phase field $\phi$. 


\section{Numerical Experiments}

In this section, we consider numerical experiments split in two groups, depending on whether or not the brittle thin sheet is adhered to a substrate. The goal of these numerical experiments is two-fold, on the one side to determine whether the variational phase-field model of fracture of thin sheets presented above is able to reproduce the phenomenology observed experimentally, and on the other side to examine the boundaries of approximate theories for tearing of thin sheets. We note that in our phase-field simulations, there is no sharp crack but rather a continuous yet localized phase-field and the deformation is continuous. However, for physical clarity, we post-process our simulations by removing elements where the phase-field is very low.
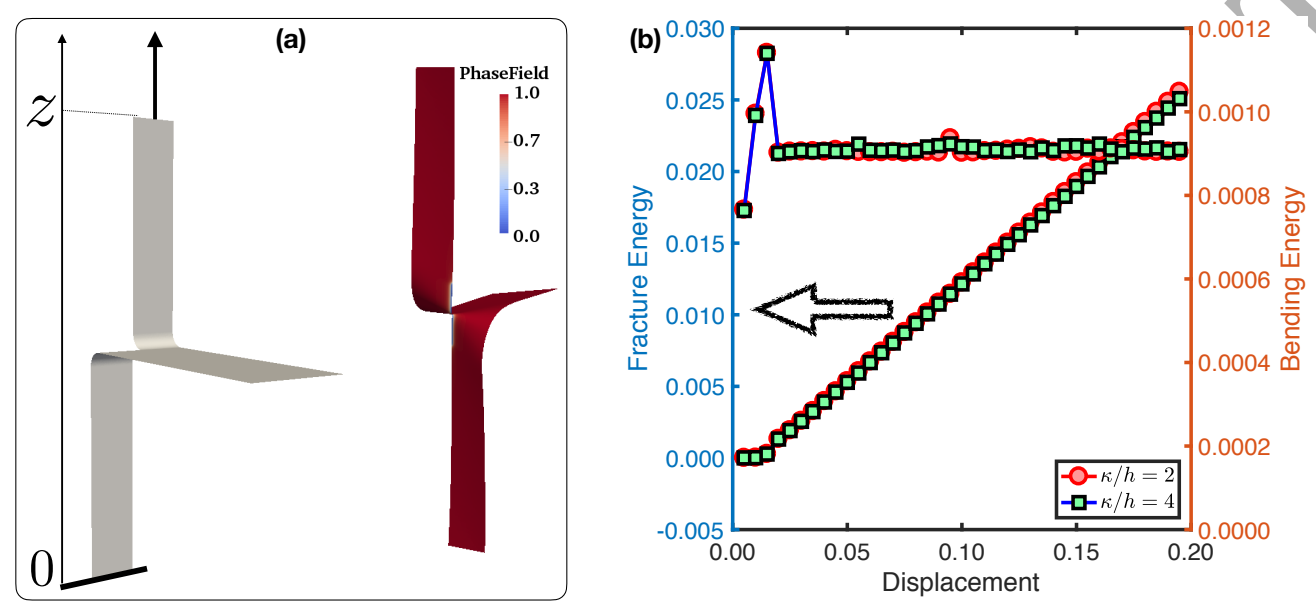

Figure 2: Numerical simulation of crack propagation in a trouser-test (a). We performed simulations with a reference mesh $(\kappa / h=2)$ and a fine mesh $(\kappa / h=4)$, obtaining very similar results in terms of bending energy and fracture energy as a function of the imposed displacement in the trouser-test (b), and justifying our criterion for mesh resolution $(\kappa / h=2)$.

\subsection{Tearing of thin sheets without adhesion}

We first considered a trouser-test in which a long thin strip with a pre-crack defining two flaps is torn apart, see Figure 2(a). In this simple test, the crack is expected to advance stably along a straight path Bayart et al. (2010), with a length proportional to the applied displacement. We used this example to examine the effect of mesh resolution. The discretization should be able to resolve the phase-field profile along a crack, characterized by the length-scale $\kappa$, which can lead to very fine meshes and expensive simulations. The material parameters are $E=1.8 \times 10^{7}, t=5 \times 10^{-4}, G_{c}=5 \times 10^{2}, \nu=0.3$ and regularization parameter is $\kappa=2 \times 10^{-3}$. The trouser-test simulation was performed at fixed $\kappa$ and with two meshes: a reference mesh satisfying $\kappa \approx 2 h$ and a finer mesh satisfying $\kappa \approx 4 h$ and leading to much longer simulation times. Figure 2(b) shows that the bending energy, which remains nearly constant during crack propagation, and the surface energy (a proxy for crack length) obtained with the two meshes agrees quite well. On the basis of this and other simulations, we performed all calculations in the remainder of the paper with meshes satisfying $\kappa \approx 2 h$, at least in the regions where cracks are expected. This mesh resolution represents a compromise between numerical accuracy and manageable computational cost. Similarly, the discretization needs to resolve the cohesive lengths, which requires that $\delta_{\mathrm{n}} \geqslant 2 h$ and $\delta_{\mathrm{t}} \geqslant 2 h$.

We also used this example to quantitatively examine the sharp interface interpretation of the fracture functional in Eq. (10), according to which $\partial \Pi_{\text {frac }} / \partial s$ should be equal to $G_{c} t$, where $d s$ is an increment in crack length. In this example, geometry imposes that $d z=2 d s$, where $d z$ is an increment in imposed displacement. This relation implies that $\partial \Pi_{\mathrm{frac}} / \partial s=2 \partial \Pi_{\mathrm{frac}} / \partial z$, which can be evaluated from the remarkably constant slope of the curve Figure 2(b). This slope is approximately equal to $0.27 / 2$, providing an estimate for the fracture surface energy very close to $G_{c} t=0.25$ used in the simulations.

We then considered a setup in which a spiral crack develops by pulling a flap perpendicularly to the thin sheet (Romero et al., 2013). We considered an annular thin sheet with traction-free boundary conditions 

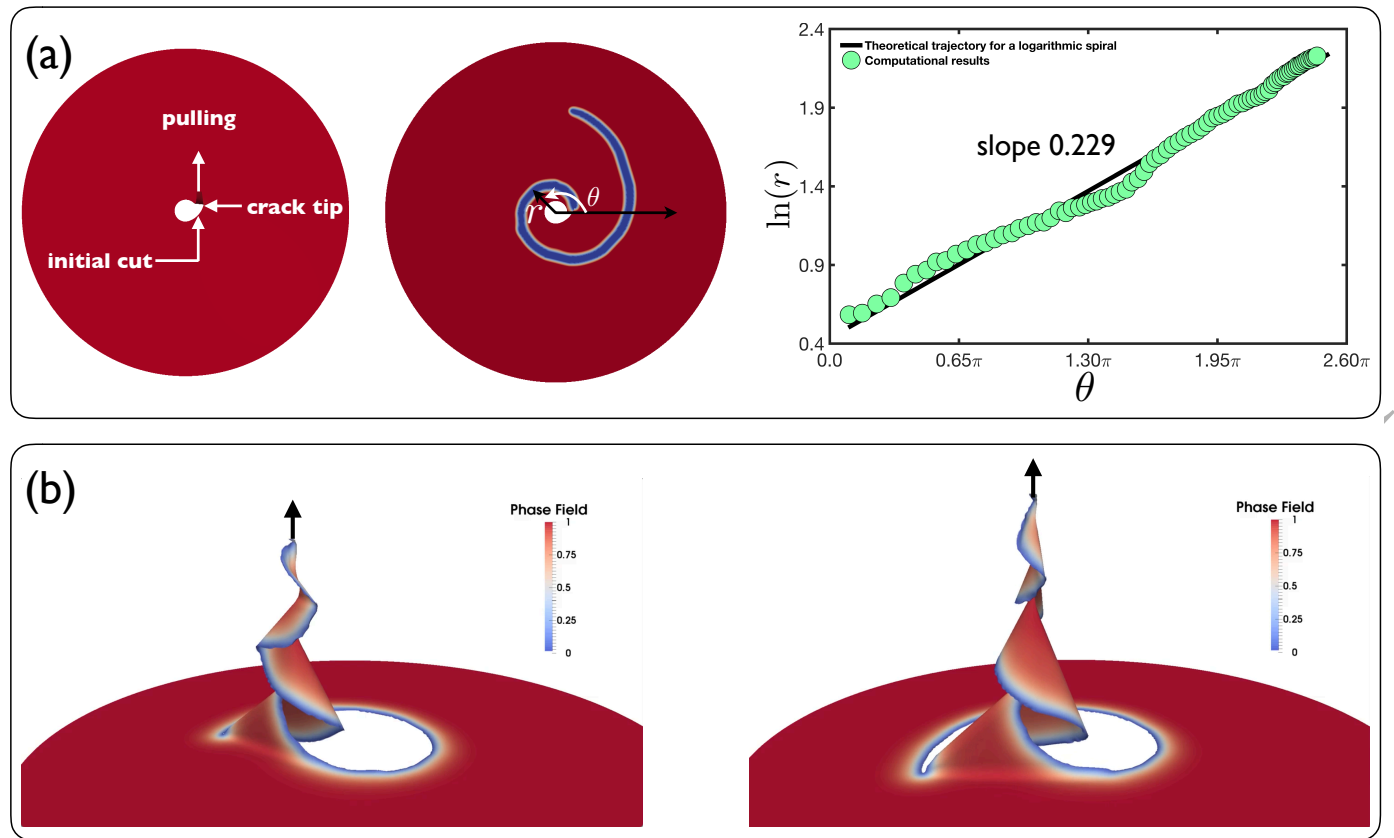

Figure 3: Spiral crack produced by pulling a flap of thin sheet.(a) The initial cut is tangent to the circular hole in the thin sheet and the flap is pulled vertically. Crack path in the undeformed configuration and fit of the crack path to a logarithmic spiral. (b) Deformed configuration obtained by the numerical simulation at different stages of fracture process.

in the circular hole located at the center and clamped boundary conditions at the outer boundary of the annulus. A small flap was formed by an initial cut tangent to the circular hole. The flap was pulled vertically to propagate the crack, see Figure 3(a). The parameter space of the problem is characterized by four nondimensional groups: $\nu, R_{\mathrm{inn}} / R_{\mathrm{out}}, t / R_{\mathrm{inm}}$, and $E t / G_{c}$, the latter two bearing more physical significance. In the calculations shown here, we considered $\nu=0.4, R_{\text {inn }} / R_{\text {out }}=0.1, t / R_{\text {inn }}=10^{-3}$, and $E t / G_{c}=10^{2}$.

The experiments performed by Romero et al. (2013) showed that as the flap was pulled vertically, it adopted a characteristic pine-tree shape while developing a spiral crack. Remarkably, our simulations captured this phenomenology, which involves very large shape changes and a complex crack path, see Figure $3(\mathrm{~b})$. The fracture process is dictated by the competition between the elastic energy (bending and stretching) concentrated at the fold connecting the flap with the planar part of the thin sheet and the fracture energy. Romero et al. (2013) showed that crack trajectories closely followed a logarithmic spiral. If this was the case, points $\left(r_{i}, \theta_{i}\right)$ along the crack should lie on a straight line when plotted in a semi-logarithmic scale. Figure 3(a) shows that this is the case in our simulations, with small fluctuations comparable to those in the experiments. The slope characterizing the shape of the logarithmic spiral is 0.23 in our simulations, in good agreement with the experimental value $0.24 \pm 0.01$ (Romero et al., 2013). We note that in these experiments, the parameters are such that $t / R_{\mathrm{inn}} \approx 10^{-2}$ and $E t / G_{c} \approx 10$.

In the third numerical experiment, we considered a long elastic thin sheet with two cracks positioned symmetrically and parallel to the center axis of the sheet, thus creating three flaps at one end of the sheet. Then, the flaps were torn apart as shown in Figure 4(a). The length and width of the thin sheet are denoted by $L$ and $W$, and the initial width of the central flap is $w_{0}$. In the simulations, we considered $\nu=0.3$, $L / W=2.6, w_{0} / W=0.3, t / w_{0}=6 \times 10^{-3}$ and $E w_{0} / G_{c}=10^{3}$. To prepare the initial configuration, we deformed the three flaps to form a $90^{\circ}$ angle with the rest of the sheet, and connected these flaps through cylindrical segments, see Figure 4(a). Then, the system was relaxed while the ends of the three flaps were fully constrained and the other end of the strip were constrained in the $z$ direction. To induce crack propagation, the outer flaps were incrementally displaced in the $-z$ direction while the inner flap was displaced in the $z$ direction by same amount. During this loading, all other degrees of freedom were 

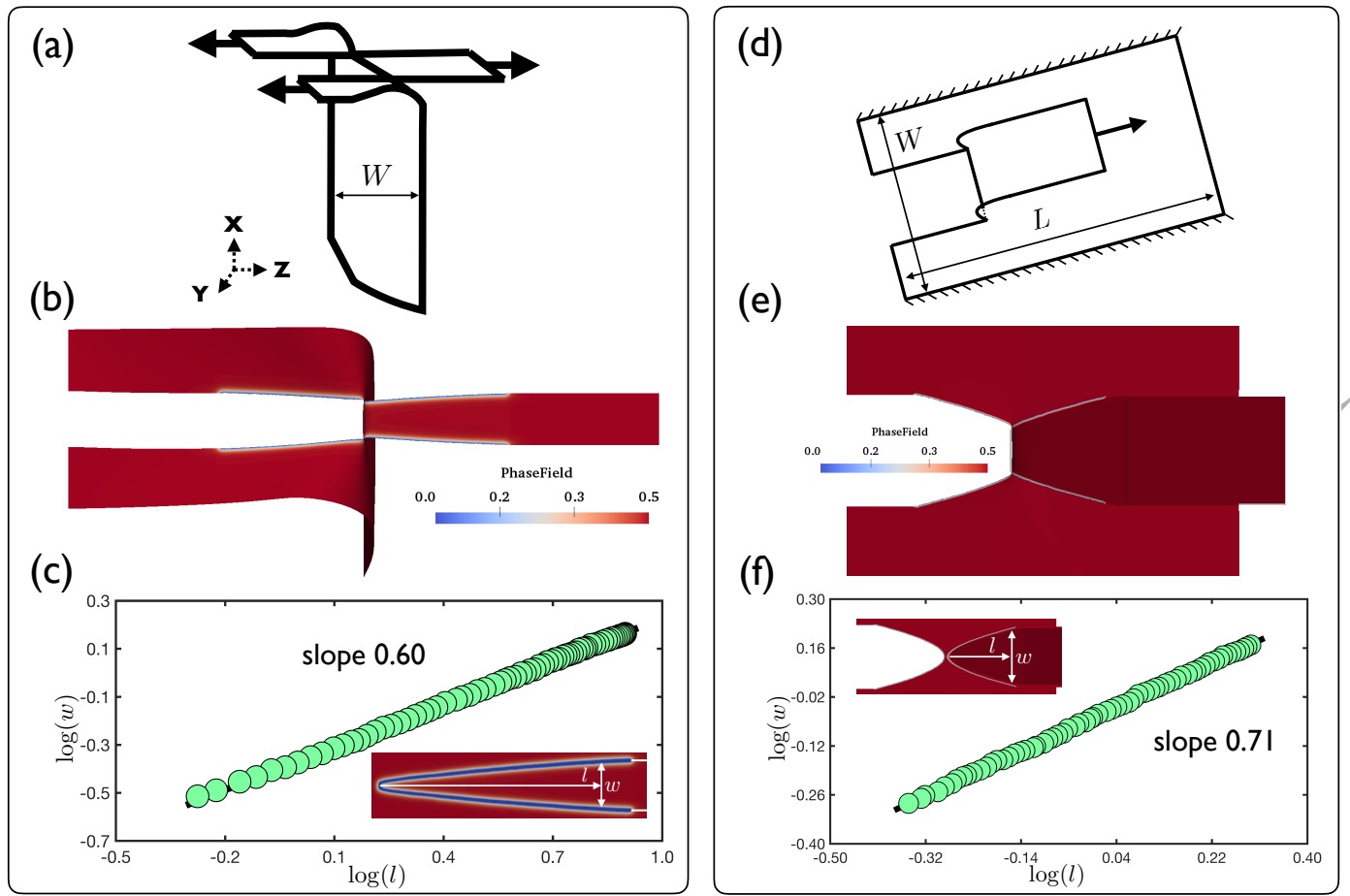

Figure 4: Tearing experiments in a three-flap configuration $(\mathrm{a}, \mathrm{b}, \mathrm{c})$ and in a laterally clamped thin sheet with a central flap $(\mathrm{d}, \mathrm{e}, \mathrm{f})$. As the convergent cracks develop, the inner flap develops a tongue-like shape (b,e). Log-log plot of the width $w$ of the center flap as a function of the distance $l$ to the tip defined by the merging point of the two cracks (c,f).

constrained and the ends of the flaps and the $z$ degrees of freedom were constrained along the bottom end of the strip.

Similarly to experimental observations, in our simulations the cracks converged as they propagate, eventually meeting and splitting the sheet into two parts. The inner flap detached from the rest of the sheet adopts a characteristic tongue-like shape as shown in Figure 4(b). We analyzed the crack trajectory by measuring the width $w$ of the inner flap as a function of the distance $l$ to the tip of the tongue. We found that $w(l)$ followed a power law with exponent 0.60 as shown in the Figure 4(c). The exponent 0.60 is in very good agreement with the exponent $0.64 \pm 0.06$ measured experimentally (Bayart et al., 2010, 2011) and with the exponent $2 / 3$ predicted by a theoretical model by Brau (2014), which combines Griffith's criterion, the maximum energy release ráte, and Euler's elastica to estimate the elastic bending energy.

In the fourth numerical experiment, we considered a peel-like experimental configuration, with the same material and geometric parameters as in the previous experiment. A thin sheet was laterally clamped. Two parallel edge cracks were initially created parallel to the center axis of the sheet, creating a flap. Then, the flap was lifted and pulled as shown in Figure 4(d) to propagate the cracks. Similarly to the previous example, we prepared the initial configuration by displacing and rotating the flap so that it remains parallel to the undeformed sheet and by connecting the displaced flap to the rest of the sheet through a half-cylinder fold. Then this configuration was relaxed keeping the lateral boundaries and the end of the flap constrained. As the flap was pulled, the distance between the end of the flap and the planar sheet was adjusted during the simulation to maintain the flap parallel to the planar sheet. This adjustment is necessary because during tearing the curvature of the fold increases as the length of the curved fold decreases.

Similarly to the previous example, the width of the flap decreased as the cracks propagate, eventually vanishing as the flap detached from the thin sheet, see Figure 4(e). The final shape of the flap is qualitatively similar to the previous simulation and can also be described by a power law of exponent 0.71 as shown in Figure 4(f). This exponent is close but noticeably differs from that obtained experimentally, $0.77 \pm 0.05$ 
by Bayart et al. (2011); Roman (2013) or theoretically, 8/11 by Brau (2014) in the unstretchable limit $E w_{0} / G_{c} \gg 1$.
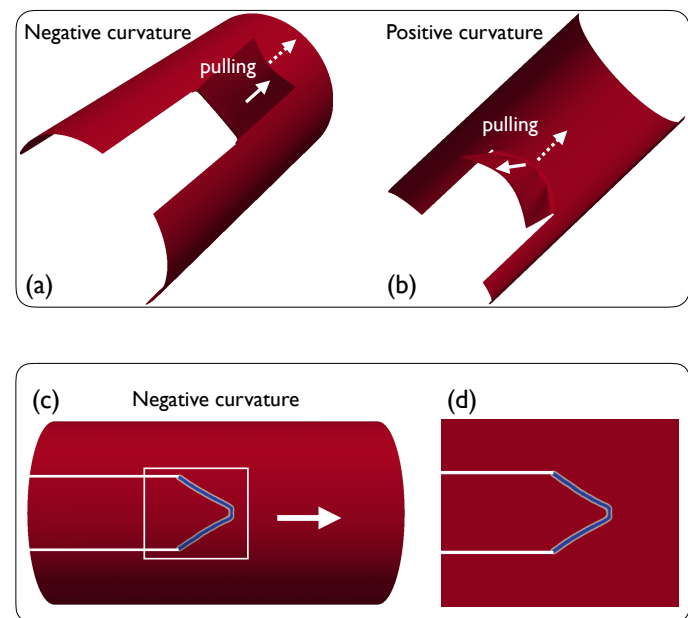

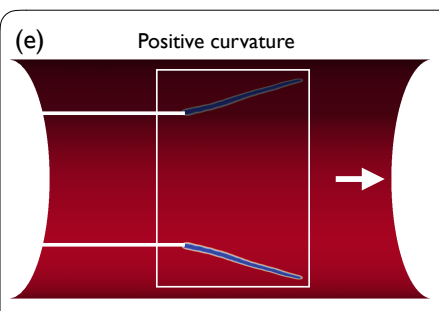

(g)

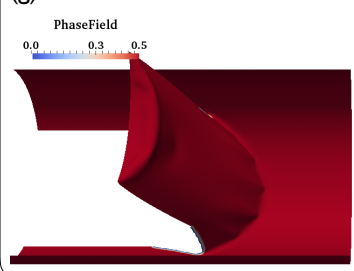

(h)

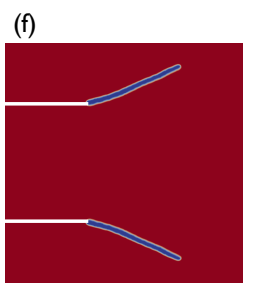

EnergyDensity

$0.0 .0 .10 .1,0.2$

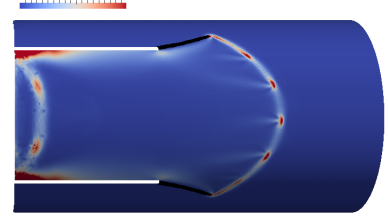

Figure 5: Tearing thin sheets adhered on cylinder substrate experiments. Tearing adhesive thin sheet on exterior side of a cylinder (negative curvature, a). Tearing adhesive thin sheet on the interior side of a cylinder (positive curvature, b). Tearing an adhesive thin sheet on the exterior side of a cylinder, converging crack path on the undeformed cylindrical configuration (c) and on the "unrolled" undeformed configurations (d). Tearing an adhesive thin sheet on the interior side of a cylinder, diverging crack path on the undeformed cylindrical configuration (e) and on the "unrolled" undeformed configurations (f). Deformed configuration during tearing $(\mathrm{g})$ and the elastic energy density in the undeformed configuration (h).

\subsection{Tearing thin sheets adhered to curved substrates}

We then considered tearing of elastic thin sheets adhered to substrates. On a flat substrate, experiments show that the detached flaps have a triangular shape (i.e. exponent 1) and hence crack trajectories are straight in this case. Before turning to flat substrates in Section 4.3, for which more quantitative results are available, we examined curved substrates. Experiments have shown that the geometry of the substrate can control the shape of the tears (Kruglova et al., 2011). The paths of the two cracks in a pulled flap are not straight and can either converge or diverge, depending on the curvature of a cylindrical substrate. We reproduced next such experiments. The initial configurations used in the simulations are shown in Figure $5(\mathrm{a}-\mathrm{b})$. Because of the curvature of the substrate, the initial and subsequent configurations are incompatible with an isometry, and therefore involve stretching. In our simulations we observed the classical motifs of paper crumpling after relaxation, such as conical point defects and stretching ridges (Witten, 2007). The flap was then pulled at constant peeling angle. The material and geometry parameters are $E=4 \cdot 10^{5}$, $\nu=0.3, G_{c}=20, \Gamma=1.0, t=10^{-3}$, a cylinder radius of 0.29 and a width of the flap of $w=0.2 / w=0.37$ for the negatively/positively curved substrate.

We first considered the negatively curved substrate and a peeling angle of $100^{\circ}$. We found that the initially parallel cracks converged and eventually the center flap detached from the substrate, as shown in Figure $5(\mathrm{c}-\mathrm{d})$. The positive curvature case with peeling angle of $70^{\circ}$ led to divergent cracks as shown in Figure 5(e-f). The convergent/divergent crack paths in the negatively/positively curved substrates observed in the simulations are in good agreement with the experimental observations by Kruglova et al. (2011). Figure 5(h) shows the elastic energy density in the shell, highlighting the d-cones and stretching ridges required to match the geometry of the adhered part of the sheet and the free-standing flap.

Our simulations of thin sheets adhered to cylindrical substrates also captured the experimentally observed deviations from "straight" crack paths, understood as geodesic curves. Indeed, upon isometric flattening of the cylindrical surfaces, crack paths did not exhibit straight trajectories, Figure 5 (f,d). To further examine the effect of substrate curvature on crack path, we performed simulations of spherical thin sheets adhered to spherical substrates, see Figure 6, which to our knowledge have not been examined experimentally. We 


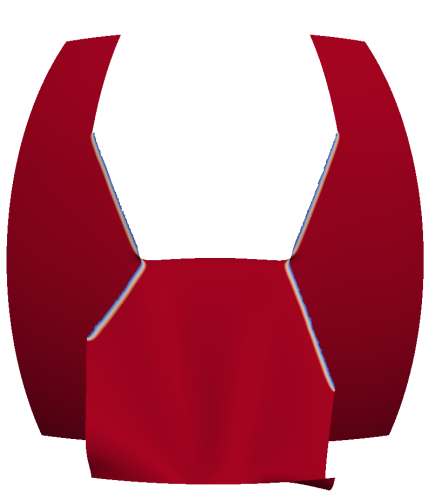

(a)
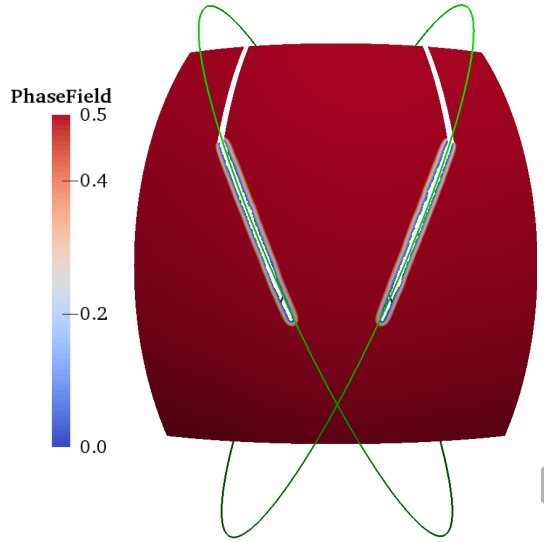

(b)

Figure 6: Tearing an adhesive thin sheet from the exterior side of a spherical substrate. Deformed configuration during tearing (a) and cracks represented in the undeformed configuration, showing that they closely follow geodesic curves shown in green (b).

found that crack paths consistently followed "straight" paths, that is great circles of the sphere, generalizing the straight converging paths observed in the case of the flat substrate (Section 4.3). Since crack paths followed geodesics on the sphere and on the plane, but not on the cylinder, we speculated that deviations from a straight path could be related to the curvature anisotropy.

(a)
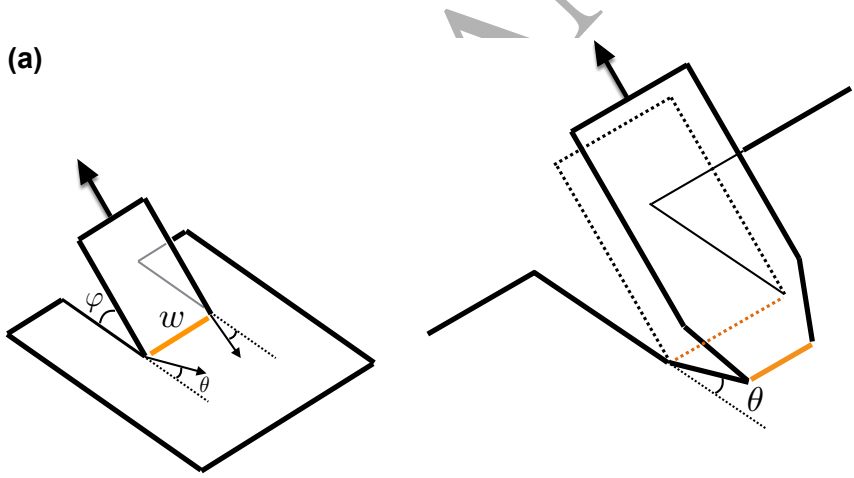

(b)

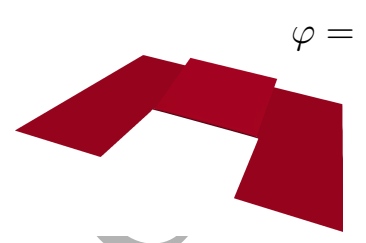

$\varphi=165^{\circ}$
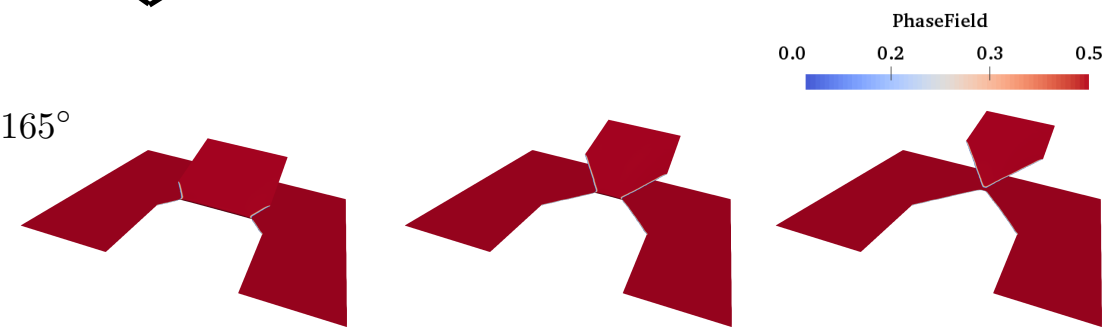

Figure 7: (a) Schematic diagram of the tearing experiments of adhesive thin sheets on a flat substrate and of the geometrical parameters necessary to describe the shape of the tear. (b) Snapshots of a tearing simulation: deformed configuration colored by the phase-field.

\subsection{Tearing thin sheets adhered to flat substrates}

We considered next an elastic thin sheet adhered to a flat substrate, in which a rectangular flap was created by cutting two paralleled cracks on one end of its edges. Before launching the simulation, we first prescribed a cylindrical segment connecting the substrate to a flap forming an angle of $\varphi$ with the planar 
substrate (see the Figure $7(\mathrm{a})$ ). The radius of curvature of the cylindrical segment was estimated as in Kruglova et al. (2011). Then, holding fixed the end of the flap, we relaxed the system. The initial length of the flap was long compared to the radius of curvature, minimizing any boundary effect. The flap was then pulled with fixed peeling angle $\varphi$, causing the initially parallel cracks to propagate inwards and the flap to progressively detach from the substrate. As in experiments, the two crack tips merged into a point, completely detaching the flap and leaving a perfectly triangular tear characterized by the angle $\theta$, Figure $7(\mathrm{~b})$.

(a)

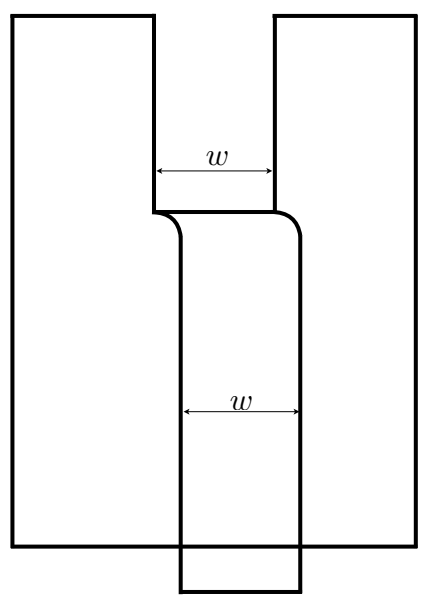

(b)

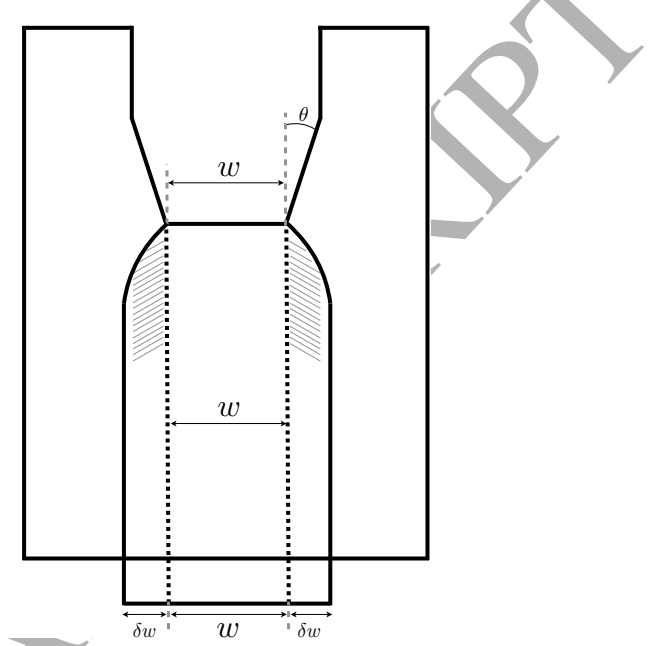

Figure 8: (a) When the cracks are parallel and the fold is singly curyed, its bending energy can be computed by multiplying the elastic energy per unit length of the optimal profile of the fold by its width $w$. (b) When the cracks converge, such a calculation ignores the bending energy in the shaded region.

The current analytical model to understand these experiments relies on three strong assumptions (Hamm et al., 2008; Roman, 2013):

(i) The system is assumed to be completely elastic, except for the fracture processes.

(ii) In the strong adhesion limit $\Gamma w \gg G_{c} t$, the fold connecting the flap and the adhered sheet is singly curved. Therefore stretching and bending do not compete, the sheet can be assumed to be inextensible, and the energy of the fold s purrely due to bending.

(iii) The mechanics of the fold is modeled using the classical Euler elastica theory to find the optimal profile for the fold and its elastic energy per unit length, assuming that the debonding front is sharp. The energy of the fold is then obtained by multiplying this elastic energy per unit length and the distance between the crack tips, i.e. the width of the strip at the detachment line. Thus, this model disregards the bending energy of a small region near the edges of the fold when $\theta \neq 0$, see Figure 8 .

An important consequence of assumption (iii) is that the elastic energy of the fold depends only on the distance between the crack tips, that is the position of the crack tips, but not their previous path. As a result, this model predicts that the system forgets its past. With these assumptions and following an energy method combining Griffith's theory with the maximum energy release rate path selection criterion, an analytical expression can be obtained for the crack path angle as (Roman, 2013)

$$
\sin \theta=\frac{\sqrt{2 \Gamma B}}{G_{c} t}\left[\frac{1-\cos (\varphi / 2)}{\sin (\varphi / 2)}\right],
$$

where $B=E t^{3} / 12\left(1-\nu^{2}\right)$ is the bending rigidity of the elastic sheet. This equation shows that the triangular shape of the tear is determined by the material constants $B, \Gamma$ and $G_{c} t$, and by the peeling angle $\varphi$. The case $\varphi=180^{\circ}$ was carefully studied experimentally in Hamm et al. (2008), where $B$ and $G_{c} t$ were varied. 
(a)
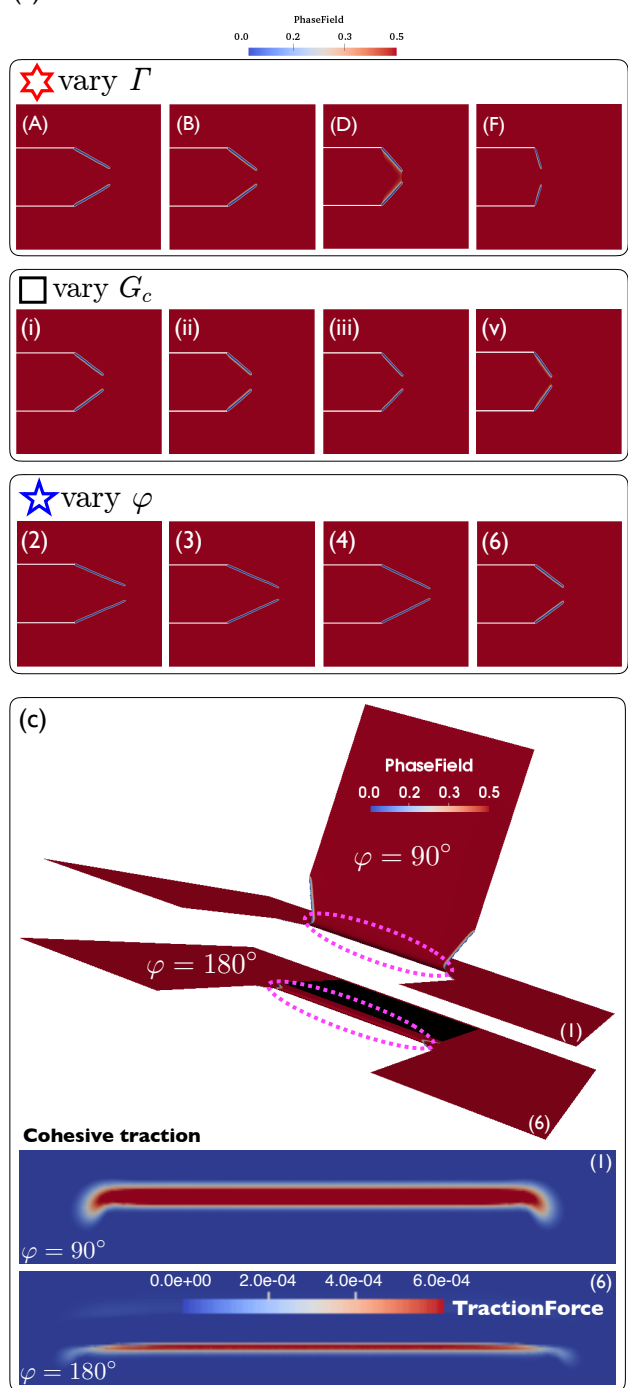

(b)

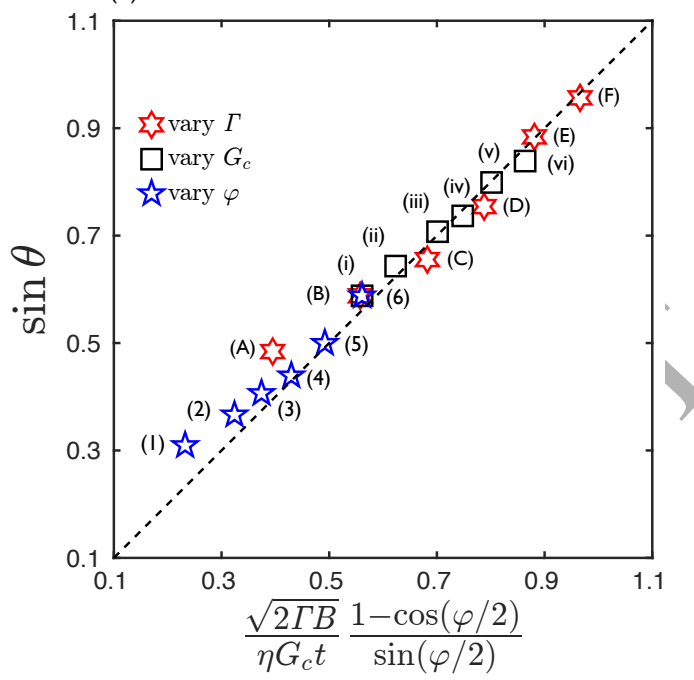

Figure 9: (a) Crack paths for numerical experiments with different material parameters. In the first row, we fix $\varphi=180^{\circ}$ and $G_{c}=20$ and vary the adhesion energy: (A) $\Gamma=0.5$, (B) $\Gamma=1.0$, (C) $\Gamma=1.5$, (D) $\Gamma=2.0,(\mathrm{E}), \Gamma=2.5$, (F) $\Gamma=3.0$. In the second row, we fix $\varphi=180^{\circ}$ and $D=1.0$ and vary the fracture energy: (i) $G_{c}=20$, (ii) $G_{c}=18$, (iii) $G_{c}=16$, (iv) $G_{c}=15$, (v) $G_{c}=14$, (vi) $G_{c}=13$. In the third row, we fix $\Gamma=1.0$ and $G_{c}=20$ and vary the peeling angle: (1) $\varphi=90^{\circ},(2) \varphi=120^{\circ}$, (3) $\varphi=135^{\circ}$, (4) $\varphi=150^{\circ}$, (5) $\varphi=165^{\circ}$, (6) $\varphi=180^{\circ}$. The crack path is represented by the phase-field in the undeformed configuration. (b) Fit of the computational data with the theoretical prediction in Eq. (19) with parameter $\eta=0.54$. (c) Map of the cohesive traction between the thin sheet and the adhesive substrate for two peeling angles. (d) Morphology of the fold between the flap and the adhered part of the thin sheet for two adhesion energies corresponding to (A) and (F).

To test this relationship, we performed tearing simulations varying various parameters but setting in all cases $E=2 \cdot 10^{5}, \nu=0.3, t=10^{-3}$ and $w=0.4$ in a square domain of unit lateral size. In a first set of simulations, we fixed the peeling angle $\varphi$ and the fracture energy $G_{c}$ and varied the surface energy $\Gamma$. In a second set of simulations, we fixed $\varphi$ and $\Gamma$, and varied $G_{c}$. In a third set of simulations, we fixed $G_{c}$ and $\Gamma$, and varied $\varphi$. The results are summarized in Figure $9(\mathrm{a}, \mathrm{b})$. In agreement with the previous theory and experiments, in all cases we found that cracks are straight, allowing us to easily measure the crack angle from the phase-field colormaps. The figure also shows that with our choices of parameters we were able to span a wide range of crack angles. As shown in Figure 9(b), our computational results agree very well 
with the theoretical prediction in Eq. (19) for nearly all calculations, although some points such as (1) or (A) exhibit significant discrepancies from the theoretical relation. To understand these discrepancies, we examined more closely these simulations.

Simulation (1) corresponds to the smallest peeling angle. Figure 9(c) shows the cohesive traction between the thin sheet and the adhesive substrate for the lowest and the highest peeling angles in our simulations. The spacial spreading of the traction is a consequence of the finite length-scales $\delta_{\mathrm{n}}$ and $\delta_{\mathrm{t}}$ in our cohesive model. Our simulations show that the width of the traction band strongly depends on the peeling angle. For a high peeling angle, this width is very narrow in closer correspondence with assumption (iii) of the theoretical model, according to which tractions are localized along a line of zero width. However, for low peeling angle this width is much larger and, presumably, the cohesive tractions significantly modify the profile of the fold. This possibility was already hypothesized by Hamm et al. (2008). Beyond the details of our cohesive model, these calculations identify a mechanism by which Eq. (19) may break down, particularly at low peeling angles.

Simulation (A) also departs from the theoretical prediction, and corresponds to the lowest adhesion energy and to the lowest non-dimensional number $\Gamma w /\left(G_{c} t\right)=10$. We recall that Eq. (19) was derived in the limit in which this number is large. Close examination of the geometry the fold in this simulation shows that it exhibits double curvature, and hence non-zero Gaussian curvature and a noticeable amount of stretching (Witten, 2007; Zhang and Arroyo, 2014, 2016), Figure 9(d). In contrast, a simulation with higher adhesion energy such as (F) exhibits a singly curved fold geometry, except in the close vicinity of the fold ends. Thus, the energetics of the fold in (A) are much more complex and depend on an interplay between adhesion, bending and stretching, which departs from assumption (ii) leading to Eq. (19).

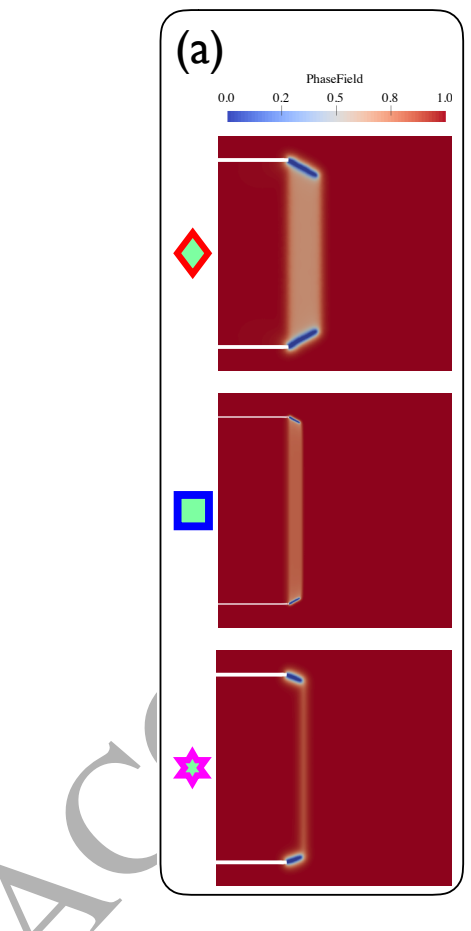

(b)

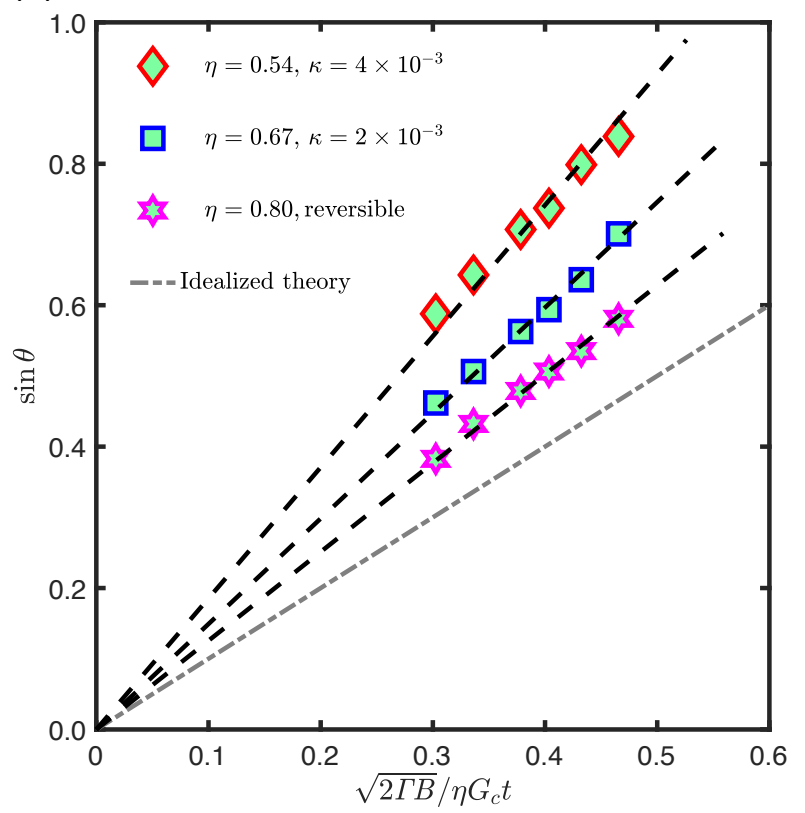

Figure 10: Examination of Eq. (19) in simulations keeping $\varphi=180^{\circ}$ and $\Gamma=1.0$ fixed and varying $G_{c}$ (i.e. $G_{c}=20,18,16$, 15, 14). (a) Phase-field represented in the undeformed configuration using a scale that better highlights lower values for our reference model (top), a model with a reduced $\kappa$ (middle) and a model allowing for damage healing (bottom). (b) Fit of the crack direction to Eq. (19) for the different models, requiring different values of the factor $\eta$, which is 1 in the ideal theoretical model.

We then turned our attention to the fact that to fit our data to Eq. (19) in Figure 9(b), we had to introduce a factor $\eta=0.54$ in the denominator or the right-hand-side of Eq. (19). To fit their experimental results, Hamm et al. (2008) also had to introduce such a factor of similar magnitude, whose origin was unclear 
although plastic deformation, observed to take place in the folded region, was suspected as a possible reason for discrepancy. We examined more closely the mechanism that explains this factor in our simulations. We noticed that, in addition to the highly localized damage along the propagating cracks, our numerical solutions exhibited a nearly homogeneous and moderate damage $(\phi \approx 0.66)$ in the region of the flap delimited by the newly created cracks, Figure 10(a), in contradiction with assumption (i) of the theoretical model. We observed that already in the preparation stage of the simulation, the fold between the flap and the adhered part of the sheet lead to partial damage co-localized with curvature, which was developed by the model to relax the bending elastic energy at the expense of a slight increase in regularized fracture energy. We reasoned that this homogeneous damage was left behind by the fold as it swept the triangular part of the flap during propagation, since the irreversibility condition precludes damage healing. This kind of coexistence between homogeneous and localized damage in phase-field models of fracture has been previouslydiscussed (May et al., 2015; Cazes and Moës, 2015).

The homogeneous damage in our phase field model of fracture is spurious and a consequence of the lack of threshold for damage evolution in this model. The spurious homogeneous damage is absent in other phasefield models of fracture (Pham et al., 2011; Pham and Marigo, 2013; Marigo et al., 2016), which require, however, more involved solution methods. Within our phase-field model, this effect can be alleviated by reducing the regularization parameter $\kappa$ (Pham et al., 2011; Borden et al., 2012; Tanné et al., 2018). To check if the factor $\eta$ was related to spurious homogeneous damage, we performed calculations with a two-fold reduction in $\kappa$, which required computational meshes twice as fine. As expected, the simulations exhibited lower homogeneous damage $(\phi \approx 0.82)$ and we could fit Eq. (19) with a factor $\eta=0.67$ closer to 1 , Figure 10(b). To further test the role of homogeneous damage, we performed simulations in which we lifted the irreversibility condition, which is unphysical but can be done in our calculations. In these simulations, the partial damage was restricted to the fold, Figure 10(a), and we could fit Eq. (19) with a factor $\eta=0.80$ even closer to 1, Figure 10(b).

Taken together, these results show that homogeneous damage taking place at the fold modifies the energy competition leading to Eq. (19) in a remarkably simple way, which only requires introducing a factor $\eta \leq 1$ in this relation. It is thus conceivable that analogous inelastic processes, e.g. plasticity, may have developed at the fold in the experiments by Hamm et al. (2008). Further theoretical work is required to more quantitatively explain tearing in the presence of multiple localized and distributed inelastic processes (Alessi et al., 2015).

\section{Conclusions}

Tearing of thin sheets is an important mode of fracture that has been extensively examined experimentally and theoretically, invoking strong assumptions difficult to control. However, this phenomenon had not been studied computationally. Here, we have developed a computational strategy to simulate brittle fracture in thin elastic sheets accounting for geometric nonlinearity and adhesion to a substrate. We have simulated a wide variety of tearing experiments of thin sheets adhered to a substrate or not. Our simulations reproduce important qualitative features of the crack paths, such as their power-law or logarithmic spiral geometry, as well as theoretical estimates for the crack path in adhered sheets based on the maximum energy release rate. Taken together, our results show that the regularized variational approach to brittle fracture naturally generalizes to fracture in thin elastic sheets, and is able to predict complex crack paths in the presence of strong geometric nonlinearity.

Importantly, our simulations have allowed us to examine the limits of current theories. We have shown how finite extensibility, finite cohesive length or irreversible mechanical processes at the fold between a torn flap and the adhered part of the thin sheet can explain deviations from the theoretical crack direction. All these phenomena involve small-scale processes in the vicinity of the fold. These bounds in our theoretical understanding of tearing of thin sheets could motivate new experiments and theories. From a modeling point of view, the phase-field model of fracture used here couples homogeneous and localized damage in a way that is difficult to control and that depends on the regularization parameter $\kappa$, which also controls the crack width. A better approach to systematically understanding tearing should be based on a model that more clearly distinguishes between the homogeneous and localized damage, for instance coupling phase-field 
models of fracture that do not exhibit distributed damage (Pham et al., 2011; Pham and Marigo, 2013) and models accounting for bending-induced damage or plasticity.

\section{Acknowledgment}

BL was supported by a labex CalsimLab fellowship/scholarship. The labex CalsimLab, reference ANR11-LABX-0037-01, is funded by the program "Investissements d'avenir" of the Agence Nationale de la Recherche, reference ANR-11-IDEX-0004-02. MA acknowledges the support of the Generalitat de Catalunya through the prize "ICREA Academia" for excellence in research and of the European Research Council (CoG-681434).

\section{Reference}

\section{References}

Abdollahi, A., Arias, I., 2012. Phase-field modeling of crack propagation in piezoelectric and ferroelectric materials with different electromechanical crack conditions. Journal of the Mechanics and Physics of Solids 60 (12), 2100-2126.

Alessi, R., Marigo, J.-J., Vidoli, S., 2015. Gradient damage models coupled with plasticity: variational formulation and main properties. Mechanics of Materials 80, 351-367.

Amiri, F., Millán, D., Arroyo, M., Silani, M., Rabczuk, T., 2016. Fourth order phase-field model for local max-ent approximants applied to crack propagation. Computer Methods in Applied Mechanics and Engineering 312, 254-275.

Amiri, F., Millán, D., Shen, Y., Rabczuk, T., Arroyo, M., 2014. Phase-field modeling of fracture in linear thin shells. Theoretical and Applied Fracture Mechanics 69, 102-109.

Baldelli, A., Bourdin, B., Marigo, J., Maurini, C., 2013. Fracture and debonding of a thin film on a stiff substrate: analytical and numerical solutions of a one-dimensional variational model. Continuum Mechanics and Thermodynamics 25 (2-4), 243-268.

Baldelli, A. L., Babadjian, J., Bourdin, B., Henao, D., Maurini, C., 2014.) A variational model for fracture and debonding of thin films under in-plane loadings. Journal of the Mechanics and Physics of Solids 70, 320-348.

Bayart, E., Boudaoud, A., Adda-Bedia, M., 2010. On the tearing of thin sheets. Engineering Fracture Mechanics 77 (11), $1849-1856$.

Bayart, E., Boudaoud, A., Adda-Bedia, M., 2011. Finite-distance singularities in the tearing of thin sheets. Physical Review Letters 106 (19), 194301.

Biermann, H., Levin, A., Zorin, D., Jul. 2000. Piecewise smooth subdivision surfaces with normal control. In: Proceedings of the 27th annual conference on Computer graphics and interactive techniques. ACM Press/Addison-Wesley Publishing Co., New York, New York, USA, pp. 113-120.

Borden, M., Verhoosel, C., Scott, M. A., Hughes, T. Landis, C., 2012. A phase-field description of dynamic brittle fracture. Computer Methods in Applied Mechanics and Engineering 217, 77-95.

Borden, M. J., Hughes, T. J. R., Landis, C. M., Verhoosel, C. V., 2014. A higher-order phase-field model for brittle fracture: Formulation and analysis within the isogeometric analysis framework. Computer Methods in Applied Mechanics and Engineering 273, 100-118.

Bourdin, B., 2007. Numerical implementation of the variational formulation for quasi-static brittle fracture. Interfaces and Free Boundaries 9 (3), 411-430.

Bourdin, B., Francfort, G. A., Marigo, J. J., 2000. Numerical experiments in revisited brittle fracture. Journal of the Mechanics and Physics of Solids 48 (4), 797-826.

Bourdin, B., Francfort, G. A., Marigo, J. J., 2008. The variational approach to fracture. Journal of Elasticity 91 (1-3), 5-148.

Bourdin, B., Marigo, J., Maurini, C., Sicsic, P., 2014. Morphogenesis and propagation of complex cracks induced by thermal shocks. Physical Review Letters 112 (1), 014301.

Braides, A., 1998. Approximation of free-discontinuity problems. Springer, Berlin.

Brau, F., 2014. Tearing of thin sheets: Cracks interacting through an elastic ridge. Physical Review E 90 (6), 062406.

Burger, M., Esposito, T., Zeppieri, C. I., 2015. Second-order edge-penalization in the Ambrosio-Tortorelli functional. Multiscale Modeling \& Simulation 13 (4), 1354-1389.

Cazes, F., Moës, N., 2015. Comparison of a phase-field model and of a thick level set model for brittle and quasi-brittle fracture. International Journal for Numerical Methods in Engineering 103 (2), 114-143.

Ciarlet, P. G., 2005. An introduction to differential geometry with applications to elasticity. Journal of Elasticity 78 (1-3), $1-215$.

Cirak, F., Long, Q., 2011. Subdivision shells with exact boundary control and non-manifold geometry. International Journal for Numerical Methods in Engineering 88 (9), 897-923.

Cirak, F., Ortiz, M., 2001. Fully $C^{1}$-conforming subdivision elements for finite deformation thin-shell analysis. International Journal for Numerical Methods in Engineering 51 (7), 813-833.

Cirak, F., Ortiz, M., Schroder, P., 2000. Subdivision surfaces: a new paradigm for thin-shell finite-element analysis. International Journal for Numerical Methods in Engineering 47 (12), 2039-2072.

Cohen, Y., Procaccia, I., 2010. Dynamics of cracks in torn thin sheets. Physical Review E 81 (6), 066103. 
Cotterell, B., 1965. On brittle fracture paths. International Journal of Fracture Mechanics 1 (2), 96-103.

Do Carmo, M., 1976. Differential geometry of curves and surfaces. Vol. 2. Prentice-Hall.

Erdogan, F., Sih, G. C., 1963. On the crack extension in plates under plane loading and transverse shear. Journal of Basic Engineering 85, 519-525.

Folias, E., 1977. Asymptotic approximations to crack problems in shells. In: Plates and shells with cracks. Springer, pp. $117-160$.

Francfort, G. A., Marigo, J. J., 1998. Revisiting brittle fracture as an energy minimization problem. Journal of the Mechanics and Physics of Solids 46 (8), 1319-1342.

Goldstein, R. V., Salganik, R. L., 1974. Brittle fracture of solids with arbitrary cracks. International Journal of Fracture 10 (4), $507-523$.

Hamm, E., Reis, P., LeBlanc, M., Roman, B., Cerda, E., 2008. Tearing as a test for mechanical characterization of thin adhesive films. Nature Materials 7 (5), 386-390.

Hui, C., Zehnder, A., Potdar, Y., 1998. Williams meets von karman: Mode coupling and nonlinearity in the fracture of thin plates. International Journal of Fracture 93 (1-4), 409-429.

Ibarra, A., Roman, B., Melo, F., 2016. Tearing path in a thin anisotropic sheet from two pulling points: Wulff's view. Soft Matter.

Kiendl, J., Ambati, M., De Lorenzis, L., Gomez, H., Reali, A., 2016. Phase-field description of brittle fracture in plates and shells. Computer Methods in Applied Mechanics and Engineering.

Kruglova, O., Brau, F., Villers, D., Damman, P., 2011. How geometry controls the tearing of adhesive thin films on curved surfaces. Physical Review Letters 107 (16), 164303.

Li, B., Peco, C., Millán, D., Arias, I., Arroyo, M., 2015. Phase-field modeling and simulation of fracture in brittle materials with strongly anisotropic surface energy. International Journal for Numerical Methods in Engineering 102 (3-4), 711-727.

Marigo, J.-J., Maurini, C., Pham, K., 2016. An overview of the modelling of fracture by gradient damage models. Meccanica, $1-22$.

Marsden, J., Hughes, T. J. R., 1983. Mathematical foundations of elasticity. Dover Publications, Inc., New York, USA.

Maurini, C., Bourdin, B., Gauthier, G., Lazarus, V., 2013. Crack patterns obtained by unidirectional drying of a colloidal suspension in a capillary tube: experiments and numerical simulations using a two-dimensional variational approach. International Journal of Fracture 1 (184), 75-91.

May, S., Vignollet, J., De Borst, R., 2015. A numerical assessment of phase-field models for brittle and cohesive fracture: $\Gamma$-convergence and stress oscillations. European Journal of Mechanics-A/Solids 52, 72-84.

Mesgarnejad, A., Bourdin, B., Khonsari, M., 2013. A variational approach to the fracture of brittle thin films subject to out-of-plane loading. Journal of the Mechanics and Physics of Solids 61 (11), 2360-2379.

Miehe, C., Hofacker, M., Welschinger, F., 2010. A phase field model for rate-independent crack propagation: Robust algorithmic implementation based on operator splits. Computer Methods in Applied Mechanics and Engineering 199 (45), 2765-2778.

Miehe, C., Schänzel, L., 2014. Phase field modeling of fracture in rubbery polymers. part I: Finite elasticity coupled with brittle failure. Journal of the Mechanics and Physics of Solids 65, 93-113.

Millán, D., Li, B., Torres-Sánchez, A., Arroyo, M., 2018. A higher-order phase-field modeling of fracture in geometrically nonlinear kirchhoff-love shells. In Preparation.

Millán, D., Rosolen, A., Arroyo, M., 2013. Nonlinear manifold learning for meshfree finite deformations thin shell analysis. International Journal for Numerical Methods in Engineering 93 (7), 685-713.

Palaniswamy, K., Knauss, W. G., 1978. On the problem of crack extension in brittle solids under general loading. In: NematNasser, S. (Ed.), Mechanics Today. Vol. 4. Pergamon Press, pp. 87-184.

Pham, K., Amor, H., Marigo, J. J., Maurini, C., 2011. Gradient damage models and their use to approximate brittle fracture. International Journal of Damage Mechanics 20 (4), 618-652.

Pham, K., Marigo, J. J., 2013. From the onset of damage to rupture: construction of responses with damage localization for a general class of gradient damage models. Continuum Mechanics and Thermodynamics 25 (2-4), 147-171.

Reinoso, J., Paggi, M. Linder, C. 2017. Phase field modeling of brittle fracture for enhanced assumed strain shells at large deformations: formulation and finite element implementation. Computational Mechanics 59 (6), 981-1001.

Roman, B., 2013. Fracture path in brittle thin sheets: a unifying review on tearing. International Journal of Fracture 182 (2), 209-237.

Romero, V., Roman, B., Hamm, E., Cerda, E., 2013. Spiral tearing of thin films. Soft Matter 9 (34), 8282-8288.

Sicsic, P., Marigo, J., Maurini, C., 2014. Initiation of a periodic array of cracks in the thermal shock problem: a gradient damage modeling. Journal of the Mechanics and Physics of Solids 63, 256-284.

Sih, G., Paris, P., Erdogan, F., 1962. Crack-tip, stress-intensity factors for plane extension and plate bending problems. Journal of Applied Mechanics 29 (2), 306-312.

Sih, G. C., 1974. Strain-energy-density factor applied to mixed mode crack problems. International Journal of Fracture 10 (3), 305-321.

Steigmann, D. J., 2013. Koiter's shell theory from the perspective of three-dimensional nonlinear elasticity. Journal of Elasticity $111(1), 91-107$.

Takei, A., Roman, B., Bico, J., Hamm, E., Melo, F., 2013. Forbidden directions for the fracture of thin anisotropic sheets: An analogy with the wulff plot. Physical Review Letters 110, 144301.

Tanné, E., Li, T., Bourdin, B., Marigo, J.-J., Maurini, C., 2018. Crack nucleation in variational phase-field models of brittle fracture. Journal of the Mechanics and Physics of Solids 110, 80-99.

Williams, M., 1961. The bending stress distribution at the base of a stationary crack. Journal of Applied Mechanics 28 (1), $78-82$. 
Wilson, Z. A., Landis, C. M., 2016. Phase-field modeling of hydraulic fracture. Journal of the Mechanics and Physics of Solids 96, 264-290.

Witten, T., 2007. Stress focusing in elastic sheets. Reviews of Modern Physics 79 (2), 643.

Wu, C. W., 1978. Maximum-energy-release-rate criterion applied to a tension-compression specimen with crack. Journal of Elasticity 8 (2), 235-257.

Xu, X. P., Needleman, A., 1994. Numerical simulations of fast crack growth in brittle solids. Journal of the Mechanics and Physics of Solids 42 (9), 1397-1434.

Zehnder, A., Viz, M. J., 2005. Fracture mechanics of thin plates and shells under combined membrane, bending, and twisting loads. Applied Mechanics Reviews 58 (1), 37-48.

Zhang, K., Arroyo, M., 2014. Understanding and strain-engineering wrinkle networks in supported graphene through simulations. Journal of the Mechanics and Physics of Solids 72, 61-74.

Zhang, K., Arroyo, M., 2016. Coexistence of wrinkles and blisters in supported graphene. Extreme Mechanics Letters 14, 23-30.

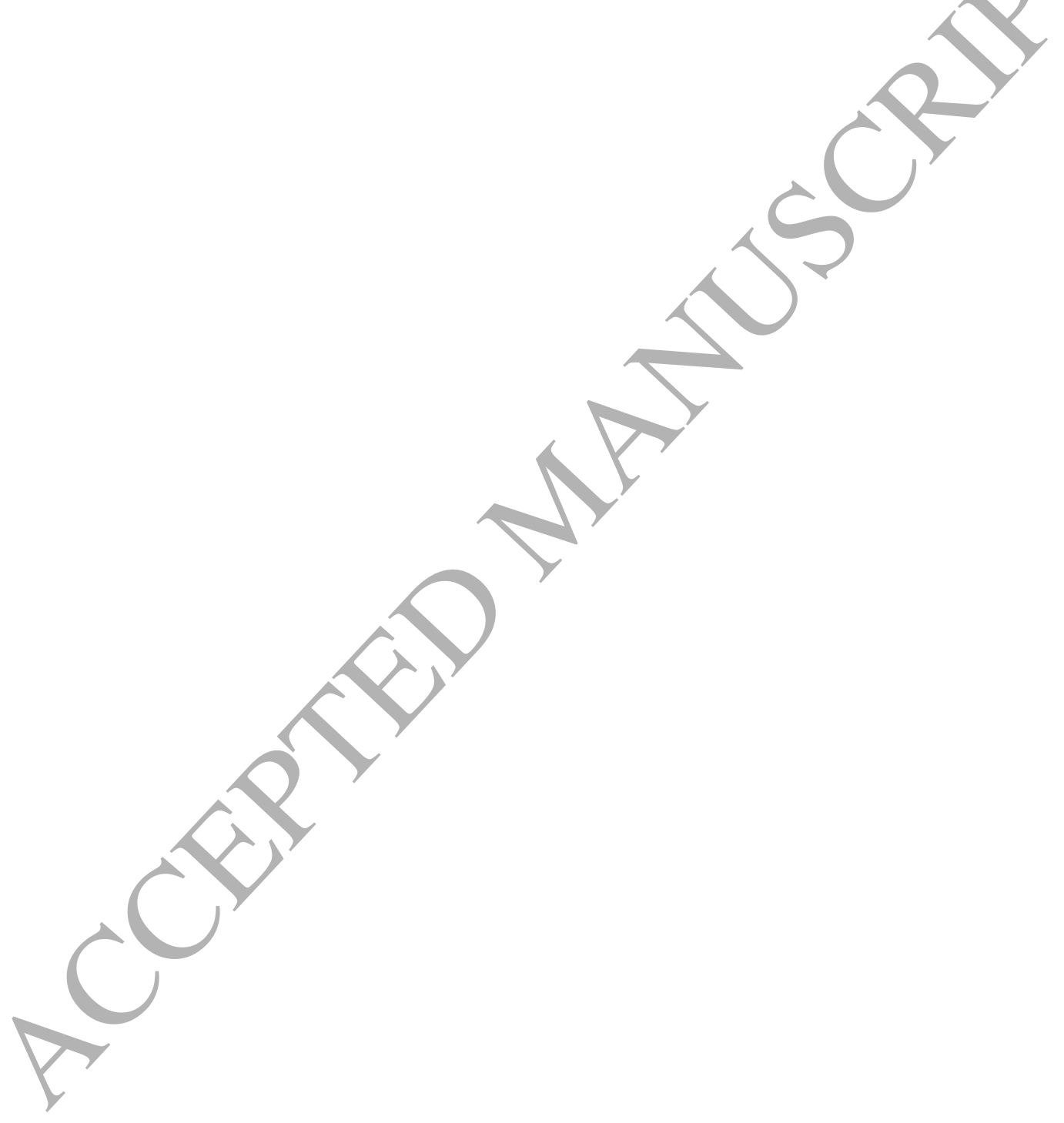

